\title{
Crying wolf in time of Corona: the strange case of ivermectin and hydroxychloroquine. Is the fear of failure withholding potential life-saving treatment from clinical use?
}

\author{
Pasquale Nardelli ${ }^{1}$, Alberto Zangrillo ${ }^{1,2}$, Gabriele Sanchini ${ }^{1}$, \\ Valery V Likhvantsev ${ }^{3,4}$, Andrey G Yavorovskiy ${ }^{4}$, \\ Carolina Soledad Romero Garcia ${ }^{5}$, Giovanni Landoni ${ }^{1,2, *}$
}

\author{
${ }^{1}$ Department of Anesthesia and Intensive Care, IRCCS San Raffaele Scientific Institute, Milan, Italy \\ ${ }^{2}$ Vita-Salute San Raffaele University, Milan, Italy \\ ${ }^{3}$ Federal Research and Clinical Center of Resuscitation and Rehabilitation, Moscow, Russia \\ ${ }^{4}$ IM Sechenov First Moscow State Medical University of the Ministry of Health of the Russian Federation (Sechenov \\ University), Moscow, Russia \\ ${ }^{5}$ Department of Anaesthesia and Critical Care, Consorcio Hospital General Universitario de Valencia, Valencia, Spain
}

\section{*Correspondence}

landoni.giovanni@hsr.it (Giovanni Landoni)

\section{Introduction}

Since the beginning of Severe Acute Respiratory Syndrome Coronavirus 2 (SARS-CoV-2) pandemic, over a hundred million people contracted the virus, putting a high pressure over hospitals and healthcare systems.

Hydroxychloroquine, lopinavir/ritonavir and azithromycin have been extensively used attempting to prevent or cure Coronavirus Disease 2019 (COVID-19) during the first wave. However, during the last few months, large clinical trials demonstrated the lack of efficacy of the abovementioned treatments [1-3].

Ivermectin is widely used to treat roundworms and ectoparasites infestation, but also has antiviral effects against RNA viruses. COVID-19 drug repurposing research focused on its possible clinical use. Ivermectin was able to inhibit SARS$\mathrm{CoV}-2$ replication in monkey kidney cell culture [4]. While its empirical use grew in Latin America, more than 45 trials investigating ivermectin in COVID-19 are ongoing all over the world.

Aim of this research letter was to understand if the administration of ivermectin in COVID-19 patients can reduce mortality.

We performed a meta-analysis of randomized clinical trials (RCTs) investigating the impact of ivermectin on mortality in COVID-19. All randomized studies reporting mortality data - comprising both published and preprints manuscripts -were included in our analysis. Two investigators independently assessed the compliance to selection criteria, selected the studies for the final analysis, and extracted data with divergences finally resolved by consensus. We followed methods previously described in details [5]. Briefly, we used Review Manager (RevMan, Version 5.4. The Cochrane Collaboration, 2020),
Mantel-Haenszel test and a fixed-effects model. The strength of the association between ivermectin administration and mortality was measured calculating the odds ratio. We focused on survival, as reported at the longest follow up available.

A total of 1323 patients were randomized in 7 RCTs performed in 6 countries. Four trials were multicentric. Ivermectin treatment resulted in a lower mortality when compared to placebo: $14 / 703(2 \%)$ vs $57 / 620(9 \%), P<0.01$, odds ratio $0.19(0.10,0.34), \mathrm{I}^{2}=13 \%$ (Fig. 1). The funnel plot was symmetrical at visual inspection. Findings were confirmed at the Influence analysis (removing one study at time).

All the mentioned RCTs included only hospitalized patients. Four trials were placebo-controlled. Ivermectin doses ranged between 12-24 mg for an average 60 kilograms subject. Duration of treatment ranged between 1 and 5 days, as reported in Table 1.

In the present meta-analysis of RCTs, administration of ivermectin reduced mortality among patients hospitalized for COVID-19. A possible rationale for these findings, other than a direct activity of ivermectin against SARS-CoV-2, [4] may involve Strongyloides hyperinfection, an uncommon complication of dexamethasone administration, [6] which is overprescribed worldwide in COVID-19 patients.

Ivermectin for the treatment of COVID-19 followed the opposite pathway of hydroxychloroquine: use of hydroxychloroquine was supported at first by medical agencies worldwide, and later proven ineffective by several RCTs including the RECOVERY Trial [1]. On the contrary, ivermectin was mostly neglected so far and only used in a few countries; nevertheless, scientific community is progressively building a body of randomized evidence which points in favor of its use. After the ruinous experience during the first wave, however, physicians became more "skeptical" and less prone to use 


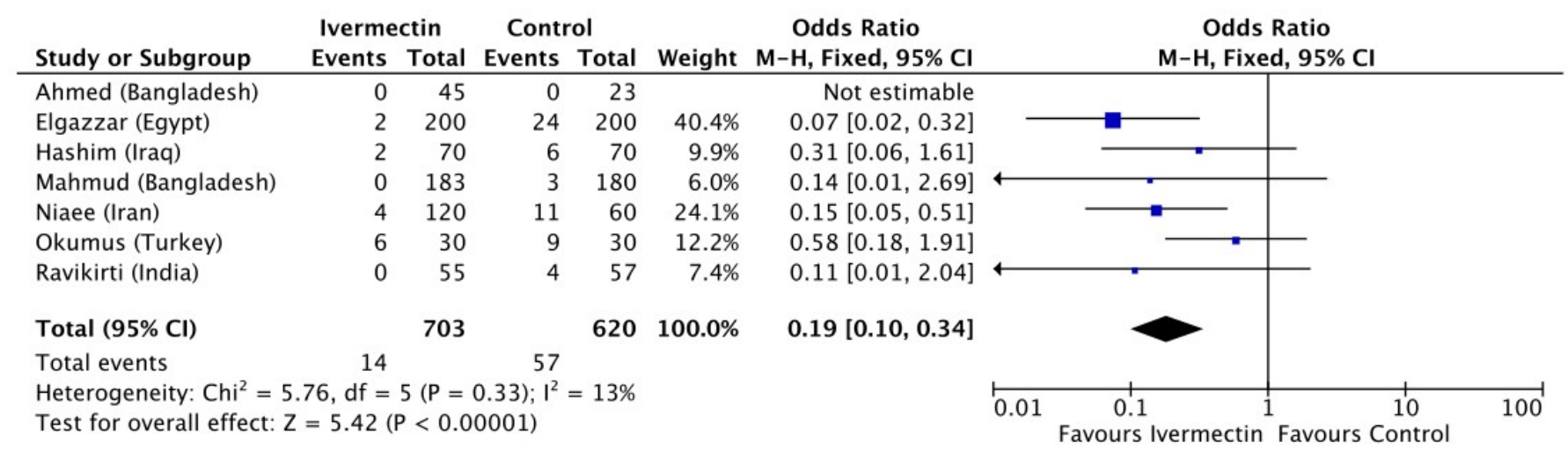

\section{F I G U R E 1. Forest plot of studies reporting mortality on ivermectin versus control in COVID-19.}

TA B L E 1. Characteristics of included studies

\begin{tabular}{|c|c|c|c|c|c|c|}
\hline Author* & Country & Disease severity & $\begin{array}{l}\text { Daily ivermectin } \\
\text { dose }\end{array}$ & $\begin{array}{l}\text { Treatment } \\
\text { duration }\end{array}$ & $\begin{array}{l}\text { Participating } \\
\text { centres }\end{array}$ & Available at \\
\hline Ahmed S & Bangladesh & Mild & $12 \mathrm{mg}$ & 5 days & 1 & Int J Infect Dis. 2020;103:214-216 \\
\hline Elgazzar A & Egypt & Mild to severe & $\begin{array}{l}400 \mathrm{mcg} / \mathrm{kg}(\max \\
24 \mathrm{mg})\end{array}$ & 4 days & 2 & $\begin{array}{l}\text { Preprint-doi.org/10.21203/rs.3.rs- } \\
\text { 100956/v1 }\end{array}$ \\
\hline Hashim HA & Iraq & Mild to severe & $200 \mathrm{mcg} / \mathrm{kg}$ & $2-3$ days & 2 & $\begin{array}{l}\text { Preprint- } \\
\text { doi.org/10.1101/2020.10.26.20219345 }\end{array}$ \\
\hline Mahmud R & Bangladesh & Mild to moderate & $12 \mathrm{mg}$ & 5 days & 1 & Clinicaltrials.gov - NCT04523831 \\
\hline Niaee MS & Iran & Mild to severe & $200-400 \mathrm{mcg} / \mathrm{kg}$ & $1-5$ days & 5 & $\begin{array}{l}\text { Preprint-doi.org/10.21203/rs.3.rs- } \\
\text { 109670/v1 }\end{array}$ \\
\hline Okumuş N & Turkey & Severe & $200 \mathrm{mcg} / \mathrm{kg}$ & 5 days & 4 & Clinicaltrials.gov-NCT04646109 \\
\hline Ravikirti RR & India & Mild to moderate & $12 \mathrm{mg}$ & 2 days & 1 & $\begin{array}{l}\text { Preprint- } \\
\text { doi.org/10.1101/2021.01.05.21249310 }\end{array}$ \\
\hline
\end{tabular}

* For data available at Clinicaltrials.gov, Responsible Party was indicated as author.

repurposed drugs in COVID-19 patients. Having cried wolf for too long may be preventing the spread of ivermectin use all over the world.

While modern medicine cannot do without ironclad evidence, in an emergency situation the use of a cheap medication without major side effects may be reasonable even if strong verification of its efficacy is still lacking. While there is an urge of large high quality RCTs, results from the reported trials all point in the same direction, and cannot be overlooked.

\section{AUTHOR CONTRIBUTIONS}

Pasquale Nardelli, Alberto Zangrillo and Giovanni Landoni designed the study. Gabriele Sanchini, Valery V Likhvantsev, Andrey G Yavorovskiy and Carolina Soledad Romero Garcia collected the data. Pasquale Nardelli and Giovanni Landoni analyzed the results and drafted the manuscript.

\section{CONFLICT OF INTEREST}

The authors declare they have no conflicts of interests.

\section{REFERENCES}

[1] Horby P, Mafham M, Linsell L, Bell JL, Staplin N, Emberson JR, et al. Effect of hydroxychloroquine in hospitalized patients with Covid-19.
New England Journal of Medicine. 2020; 383: 2030-2040.

[2] Lopinavir-ritonavir in patients admitted to hospital with COVID-19 (RECOVERY): a randomised, controlled, open-label, platform trial. Lancet. 2020; 396: 1345-1352.

[3] Furtado RHM, Berwanger O, Fonseca HA, Corrêa TD, Ferraz LR, Lapa $\mathrm{MG}$, et al. Azithromycin in addition to standard of care versus standard of care alone in the treatment of patients admitted to the hospital with severe COVID-19 in Brazil (COALITION II): a randomised clinical trial. Lancet. 2020; 396: 959-967.

[4] Caly L, Druce JD, Catton MG, Jans DA, Wagstaff KM. The FDAapproved drug ivermectin inhibits the replication of SARS-CoV-2 in vitro. Antiviral Research. 2020; 178: 104787.

[5] Bove T, Belletti A, Putzu A, Pappacena S, Denaro G, Landoni G, et al. Intermittent furosemide administration in patients with or at risk for acute kidney injury: Meta-analysis of randomized trials. PLoS ONE. 2018; 13: e0196088.

[6] Stauffer WM, Alpern JD, Walker PF. COVID-19 and Dexamethasone. Journal of the American Medical Association. 2020; 324: 623.

How to cite this article: Pasquale Nardelli, Alberto Zangrillo, Gabriele Sanchini, Valery V Likhvantsev, Andrey G Yavorovskiy, Carolina Soledad Romero Garcia, et al. Crying wolf in time of Corona: the strange case of ivermectin and hydroxychloroquine. Is the fear of failure withholding potential life-saving treatment from clinical use? Signa Vitae. 2021;17(3):3-4. doi:10.22514/sv.2021.043. 